Research Article

\title{
Decision Support System to Risk Stratification in the Acute Coronary Syndrome Using Fuzzy Logic
}

\author{
Hui Xiao $\mathbb{D}^{1},{ }^{1}$ Shah Nazir $\left(\mathbb{D},{ }^{2}\right.$ Hanmin Li, ${ }^{1}$ Habib Ullah Khan $\mathbb{D}^{3},{ }^{3}$ and Chengwei $\mathrm{Li}^{1}$ \\ ${ }^{1}$ Information Center, Zhongnan Hospital of Wuhan University, Wuhan 430071, China \\ ${ }^{2}$ Department of Computer Science, University of Swabi, Swabi, Pakistan \\ ${ }^{3}$ Department of Accounting and Information Systems, College of Business and Economics, Qatar University, Doha, Qatar \\ Correspondence should be addressed to Hui Xiao; znhospitalxh@163.com and Shah Nazir; snshahnzr@gmail.com
}

Received 18 June 2021; Accepted 19 July 2021; Published 27 July 2021

Academic Editor: Zhu Xiao

Copyright () 2021 Hui Xiao et al. This is an open access article distributed under the Creative Commons Attribution License, which permits unrestricted use, distribution, and reproduction in any medium, provided the original work is properly cited.

Acute coronary syndrome (ACS) is a set of symptoms and signs which define a range of conditions related with the unexpected reduced blood flow to the heart. In ACS, the heart muscles cannot function properly due to the decrease of blood flow. Myocardial infarction (MI) is a condition which comes under the umbrella of acute coronary syndrome. The aim of risk stratification (RS) in ACS is to recognize patients at high risk of ischemic events. Yet, no investigative study is available to identify the patients at high risk. Therefore, to facilitate this process, it would be ideal to have a reliable and trustworthy method by the help of which the doctors can make early and easy decisions for the patient and for detecting the related disease. This research used the features of GRACE Score to RS in the ACS and presented decision support system (DSS). The concept of probabilistic approach has been used as a tool to model the identified features for decision-making (DM). This technique can be further used for DM purposes to RS in the ACS in healthcare. Furthermore, the result of the proposed method has proved closer and more reliable DM of patient and then eventually can be used for advice of medicine and rest accordingly by the doctors.

\section{Introduction}

The emergency department is mostly facing the patient of chest pain. One of the main reasons for chest pain is the ACS which occurs in both short-term and long-term adverse prognosis. ACS is a set of symptoms and signs which describe conditions range connected with reduced flow of blood to heart. In ACS, heart muscles are unable to function properly due to decrease in blood flow. The aim of RS in ACS is to identify the patients at high risk of ischemic events.

According to the report of WHO [1], the cardiovascular disease is one of the top causes of death. In the admissions of hospital, the patients of acute chest pain are a big burden. This range is presented as the number present of $40 \%$ of acute medical admissions and 5\% of all emergency [2]. On time and efficient treatment is a necessary part to ensure the cost-effective and precise management of the patient. Meanwhile, the poor identification and treatments are linked with increased mortality, morbidity, and cost [3]. The identification of ACS relies on account of ischemic symptoms, such as the ischemic ECG changes, chest pain, and elevated cardiac biomarkers. There are diverse causes of ACS and the identification of MI is categorized based on the causes [4].

ACS is a multifactorial disease which includes the genetic factor caused by coronary artery obstruction by atheroma. It is influenced by other factors of risk such as diabetes, hypertension, obesity, smoking, and dyslipidemia. Among these, the hypertension and dyslipidemia are considered as the most common factors in the ACS patients, with a suggestion that preventing these factors can put in a decrease in the process of atherosclerosis. Diagnosis of patients with high risk soon after ACS has been a challenging issue for the clinicians for a few decades. Several studies exist which show that more rigorous management of the patient results in considerably good outcomes in the adverse cardiac events. The diagnosis of severity risk of ACS patient is significant, as the patient can get benefit with early and proper treatment. 
Lin et al. [5] presented a study containing a dataset of 22 million enrollees in Taiwan from 1999 to 2010 . The study has acknowledged 27948 patients with CS and sex, age, and comorbidity matched control. Multivariate regression analysis after amendment for probable cardiovascular confounders and odd ratios was calculated with 95\% confidence interval to measure the relationship between CS and ACS.

The aim of the ACS risk score is to diagnose the patient with high risk who can be managed with optimum treatment in short time. The patients of ST elevation MI and non-ST elevation MI have been subjected to the RS studies and several risk scores have been proposed. Some of the scores were tested in clinical trial populations, while others were derived from large registries.

Currently, no exploratory study is available to recognize the patients with high risk. To assist this progression, it would be ultimate to have a reliable and trustworthy method through which the practitioners can make early and easy decision for the patient. The proposed study's contribution is to use the features of GRACE Score to RS in the ACS for DM. A probabilistic approach of fuzzy tool has been used to model the feature for DM. The technique can be further used for DM purpose to RS in the ACS in healthcare. Fuzzy set is an effectual tool to convey uncertain and imperfect cognitive information with the membership, nonmembership, and hesitance degrees [6]. The fuzzy logic has a wide range of applications in various areas of research [7]. Technology acceptance model was presented through fuzzy multicriteria DM approach [8]. The study has analysed 136 research articles regarding the software development organization for cloud based technology implementation.

This paper is organised as follows: Section 2 describes works related to RS in the patients of ACS. Section 3 describes the methodology used for the planned study. Section 4 depicts the results and discussions, while the paper concludes with Section 5.

\section{Related Work}

Early DM of patient has immense prospective for refining the value of care, decreasing the care cost, and dropping error and waste. Several studies were related to RS of patient for ACS. Singh and Guttag [9] presented an algorithm to hold class imbalance in medical datasets and assessed the nonsymmetric based classification tree algorithm performance. The performance of the said algorithm is evaluated and compared with those of SVM based classifiers applied to 4219 non-ST elevation ACS patients. Three strategies were used for management class imbalance. These strategies were synthetic minority oversampling, cost-sensitive SVM learning, and random majority undersampling. Khalil et al. [10] assessed the validity and accuracy of Canadian-ACS risk score as a prognostic score for the patient in hospital with RS with ACS as compared to TIMI and GRACE risk scores in patients of Egypt. The authors of [11] assessed the clinical utility of H-FABP for suspected ACS. The H-FABP demonstrated the added value to cardiac troponin in the initial stage after onset of symptoms. The H-FABP also detected the patients at enlarged risk for upcoming actions of cardiac diseases. J. Přeček et al. [12] demonstrated a summary of patients with ACS of prognostic stratification according to baseline levels of renal function biomarkers. The authors of [13] provided a model with interpretable representation of long-term ECG recording and discovered the associations among all short heart rate patterns across the population of patients. The experiment was performed on 4557 patients with non-ST elevation ACS and confirmed that heart rate topic models considerably get better RS.

Macdonald et al. [14] compared the two clinical scoring methods of RS of suspected ACS in the emergency department. Cullen et al. [15] performed a diagnostic study in the emergency department for the patient with symptoms of ACS with two-hour sensitive Troponin Assay Results. The results were elaborated using standard measures of test accuracy. The authors of [16] reviewed up-to-date methods in the emergency care for the identification and RS of NSTEACS. Further, the authors highlighted the limitations of the existing approaches and suggested the improvements in them. An approach was presented which quantified the level to which subtle ECG morphology changes from beat to beat. The time and shape characteristics were considered as morphological variability to measure the changes in these variables in the cardiac events in sequential pairs of heart beats. The proposed method tested 400 patients of ACS [17]. The research explored the challenges that manipulate the dietary choices from patient attending a secondary prevention clinic following MI [18].

Dellas et al. [19] studied the predictive performance of Heart-type Fatty Acid Binding Protein and integrated it into the ESC algorithm. A subanalysis study was presented for the disease of diabetes and peripheral arterial disease in the patient of RS admitted after ACS [20]. Hill et al. [21] presented a roadmap for computational cardiology. The approach consisted of three stages: (i) development of baseline model, (ii) disease simulation, and (iii) translation. The authors of [22] presented that there were some barriers in the RS appropriate implementation with ACS, so they proposed developing a simple and early score for RS of the ACS patient. The study was conducted to diagnose the risk factors for the ACS for the age below 40 years in the Slemani Cardiac Hospital, Sulaimaniyah, Iraq [23]. Pezawas et al. [24] presented a study on the risk of arrhythmic death in ischemic heart disease. The study was conducted for prospective, controlled, observer-blind RS for 10 years. Lin et al. [5] presented a study for the quantification of the relationship between cervical spondylosis and ACS. Details of some of the existing approaches are shown in Table 1. These approaches along with the publication year are given.

\section{Methodology}

ACS is a set of symptoms and signs which describe variation of conditions associated with the sudden, reduced blood flow to the heart. In ACS, the muscles of the heart are unable to function appropriately due to the reduction of blood flow. MI is a situation under the umbrella of ACS. The proposed research work uses the features of GRACE Score to RS in the 
TABLE 1: Some of the existing approaches for RS.

\begin{tabular}{|c|c|c|c|c|}
\hline $\begin{array}{l}\text { S. } \\
\text { no. }\end{array}$ & Reference & Method used & Paper & $\begin{array}{c}\text { Year of } \\
\text { paper }\end{array}$ \\
\hline 1 & {$[25]$} & Coronary disease-ACS: RS & Report & 2000 \\
\hline 2 & {$[17]$} & RS following ACS through electrocardiography for measuring variability in morphology & Conference & 2008 \\
\hline 3 & {$[26]$} & Rapid RS in suspected ACS using serial multiple cardiac biomarkers: a pilot study & Journal & 2008 \\
\hline 4 & {$[20]$} & $\begin{array}{l}\text { Additive value of diabetes and peripheral arterial disease in the RS of patients admitted after an } \\
\qquad \text { ACS: a subanalysis of the PAMISCA study }\end{array}$ & Journal & 2009 \\
\hline 5 & {$[14]$} & Comparison of two clinical scoring systems for emergency department RS of suspected ACS & Journal & 2011 \\
\hline 6 & [9] & $\begin{array}{l}\text { A comparison of nonsymmetric entropy-based classification trees and support vector machine } \\
\text { for cardiovascular RS }\end{array}$ & Conference & 2011 \\
\hline 7 & {$[13]$} & Cardiovascular RS with heart rate topics & Conference & 2012 \\
\hline 8 & {$[15]$} & Performance of RS for ACS with two-hour sensitive Troponin Assay Results & Journal & 2014 \\
\hline 9 & {$[16]$} & RS in non-ST elevation ACS: risk scores, biomarkers, and clinical judgment & Journal & 2015 \\
\hline 10 & {$[21]$} & $\begin{array}{c}\text { Computational cardiology and RS for sudden cardiac death: one of the grand challenges for } \\
\text { cardiology in the } 21 \text { st century }\end{array}$ & Journal & 2016 \\
\hline 11 & {$[27]$} & RS in ACS: graced by a new score? & Journal & 2017 \\
\hline 12 & {$[28]$} & RS after ACS: scores, scores, and yet another score & Journal & 2017 \\
\hline 13 & {$[24]$} & $\begin{array}{c}\text { Risk of arrhythmic death in ischemic heart disease: a prospective, controlled, observer-blind } \\
\text { RS over } 10 \text { years }\end{array}$ & Journal & 2017 \\
\hline 14 & {$[5]$} & Risk of ACS in patients with cervical spondylosis & Journal & 2018 \\
\hline 15 & {$[23]$} & Risk factors for ACS in patients below the age of 40 years & Journal & 2018 \\
\hline 16 & {$[22]$} & ProACS risk score: an early and simple score for RS of patients with ACS & Journal & 2017 \\
\hline 18 & [29] & A new RS tool for women with ACS & Journal & 2018 \\
\hline 19 & {$[19]$} & RS of acute pulmonary embolism based on clinical parameters, $\mathrm{H}$-FABP, and multidetector CT & Journal & 2018 \\
\hline
\end{tabular}

ACS. The concept of probabilistic approach (fuzzy tool) has been used to model the identified feature for DM. The following subsections briefly describe the details of the proposed method.

3.1. RS in the ACS. The aim of RS in ACS is to identify the patients at high risk of ischemic events. Early DM in healthcare system has massive potential for refining the quality of care and reducing the cost of care, waste, and error. The risk score of Global Registry of Acute Coronary Events (GRACE) is a tool of validated RS which has incremental predictive value for RS compared with the evaluation of clinical testing [16]. Some countries have partial implementation of GRACE Score in their emergency medicine. The age factor formulates an exponential input to the GRACE Score. The addition of new cardiac biomarkers into this identification pathway can allocate to early treatment stratification. The advantages of the strong GRACE Score $[30,31]$ are based on large registry, but the disadvantage lies in including variables at the time of existing admission (laboratory results), so it is not applicable for instant stratification. The related studies to RS are discussed in Section 2 of the paper.

3.2. Fuzzy Logic. The fuzzy logic (FL) is a mathematical tool used to resolve circumstances of vagueness and uncertainty. It was developed by Zadeh in 1965 [32, 33]. FL converts the specific analysis, deals with vague information, and offers a best decision in the presence of the vague and incomplete data. Some of the applications of FL are in control system, car transmission system, washing machines, vacuum cleaner, and software system [7, 34-37]. Details concerning fuzzy idea can be found in the study in [32]. Messaoud et al. [38] used fuzzy logic for the application of cognitively stimulated use of speech information and to enhance microphone system. Liu and Zhang [39] integrated the picture fuzzy set and the linguistic term; the study has defined the picture fuzzy linguistic set (PFLS) and operations of picture fuzzy linguistic numbers for developing the Archimedean picture fuzzy linguistic weighted arithmetic averaging operator and presented various properties and special cases of this operator. They also proposed a method which deals with problem of multiattribute group DM on the basis of the developed A-PFLWAA operator. The reason behind the fuzzy logic used in the proposed is that it works in the situation of uncertainty and vagueness, while the other methods like AHP and ANP work in complex situations.

3.3. DSS for RS in ACS. Fuzzy logic is used as a DSS for the RS of the ACS. Liu et al. [40] extended the ensemble-based scoring system by using a fast extreme learning machine algorithm for neural networks. The patients of emergency department of Singapore General Hospital were examined and the features of 12-lead ECG parameters, heart rate variability, and vital signs were extracted. An ensemblebased scoring system-extreme learning machine (ESS-ELM) algorithm was proposed for the prediction of cardiac events. The features of GRACE Score are considered to model the proposed DSS. Figure 1 shows the European Society of Cardiology (ESC) rapid rule-out of ACS with hs-cTn algorithm [41]. Rapid rule-out of ACS with high-sensitivity troponin. Grace $=$ Global Registry of Acute Coronary Events; ULN = upper limit of normal, 99th percentile of 


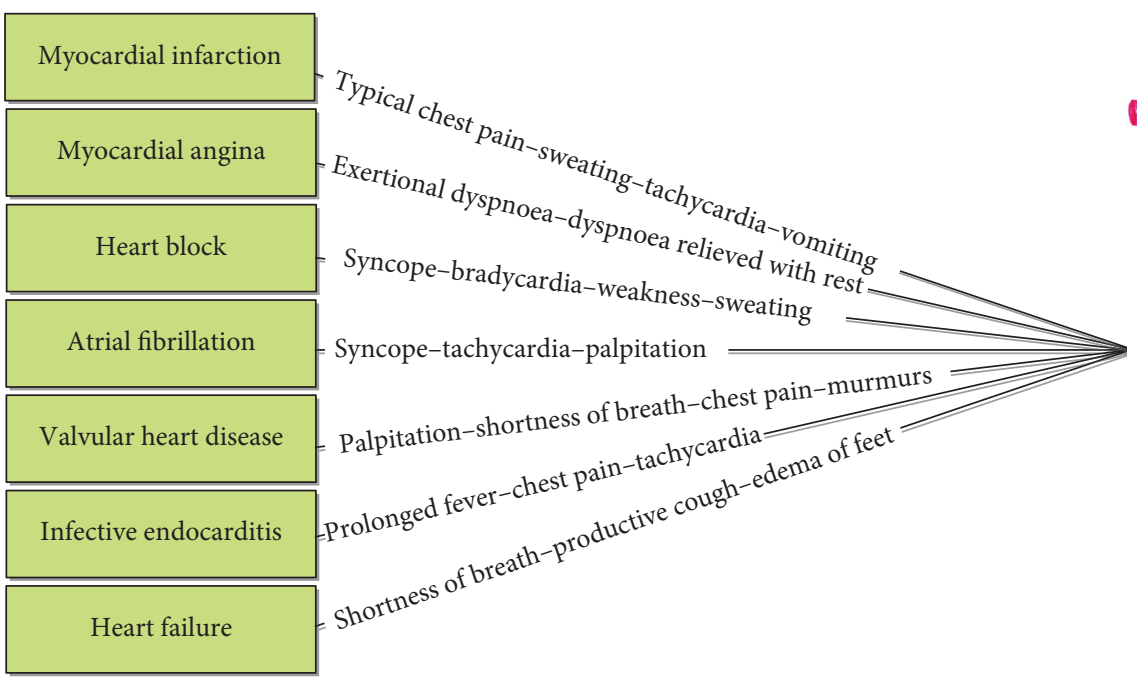

Figure 1: Graphical representation of heart diseases and their symptoms.

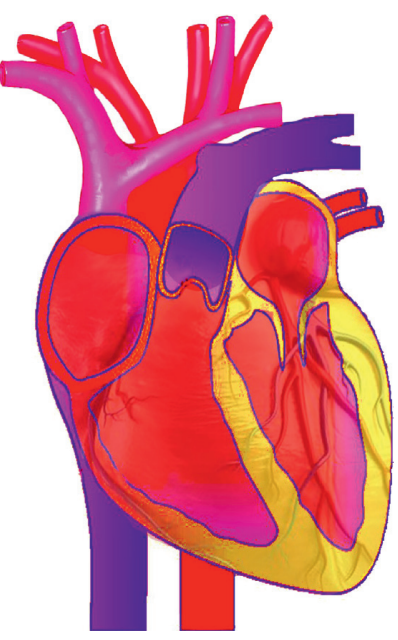

Heart disease healthy controls; hsTn = high-sensitivity troponin. Figure 1 shows the graphical representation of heart diseases and their symptoms.

The GRACE 1.0 Score is broadly certified [41]. This score diagnoses the patient in hospital as a 6-month risk of death by using the following factors: heart rate, age, Killip class, systolic BP, creatinine, elevated cTn, ST-segment deviation, and cardiac arrest. The new GRACE 2.0 Score revised GRACE algorithms for diagnosis of death at 1 and 3 years and is termed at medical device (available at http://www. gracescore.org/WebSite/WebVersion.aspx). Figure 2 shows the GRACE score, which consists of the factors age, heart rate, systolic BP, Killip class, and creatinine [16].

Figure 3 shows the generic process of fuzzification and defuzzification modeling for the proposed study of RS for ACS. The figure consists of fuzzification, database, rule base, defuzzification, and output. Once the rules are designed based on the membership function, they can be stored in database provided by the fuzzy inference system. After that, the inputs can be passed from the designed model and the output will be displayed.

Membership functions are designed individually for each input based on the score provided in the GRACE Score table. The MFs are the degree of representations of each input. The starting and ending boundaries of a particular input can be shown. All the inputs are categorized into four membership functions. The reason behind this is that some of the inputs have fewer features, while some of them are having more detailed features. So, to make uniformity in the model, all inputs were equally categorized into four membership functions. These categories show the level and severity of a specific input. Figure 4 shows the memberships functions designed for the input age.

The rest of the membership functions for the other inputs can be drawn the same as Figure 4 . The same can be plotted in the same way for the remaining membership functions.
Different rules were developed according to the defined membership functions. For understanding of the reader, some rules are given as follows:

(i) If Age (years) is 40, heart rate (bpm) is 70, systolic BP $(\mathrm{mmHg})$ is <200, and Killip class is Class I) and creatinine $(\mathrm{mg} / \mathrm{dL})$ is $0.0-0.39$, then risk stratification is very low (0.1)

(ii) If Age (years) is 49-59, heart rate (bpm) is 89-109, systolic BP $(\mathrm{mmHg})$ is 199-140, Killip class is ClassI, and creatinine $(\mathrm{mg} / \mathrm{dL})$ is $0.4-1.19$, then risk stratification is low (0.3)

(iii) If Age (years) is 69-89, heart rate (bpm) is 110-199), systolic BP $(\mathrm{mmHg})$ is 139-80, Killip class is ClassI, and creatinine $(m g / d L)$ is 1.2-3.99; then risk stratification is medium (0.5)

(iv) If Age (years) is 69-89, heart rate (bpm) is 110-199, systolic BP ( $\mathrm{mmHg})$ is 139-80, (Killip class is Clas$s I I)$, and creatinine $(m g / d L)$ is $1.2-3.99$, then risk stratification is high (0.8)

(v) If Age (years) is $\leq 90$, heart rate (bpm) is <200), systolic BP $(\mathrm{mmHg})$ is $>80$, Killip class is Class $I V$, and creatinine $(\mathrm{mg} / \mathrm{dL})$ is $>4$, then risk stratification is very high) (1) ... and so on

The proposed system is based on five inputs (age, Killip class, heart rate, systolic BP, and creatinine); rules and their output are shown in Figure 5. This system was designed in Matlab to deal with the situation of uncertainty and vagueness arising for the RS in the ACS. In this system, initially the membership functions are defined followed by the rules development from the MF, and the rules are combined to form the model. Inputs can be passed through the model and resulted output will be used to facilitate patient accordingly.

Figure 6 depicts the nomenclature of the proposed inputs, membership functions, rules, and output. The 


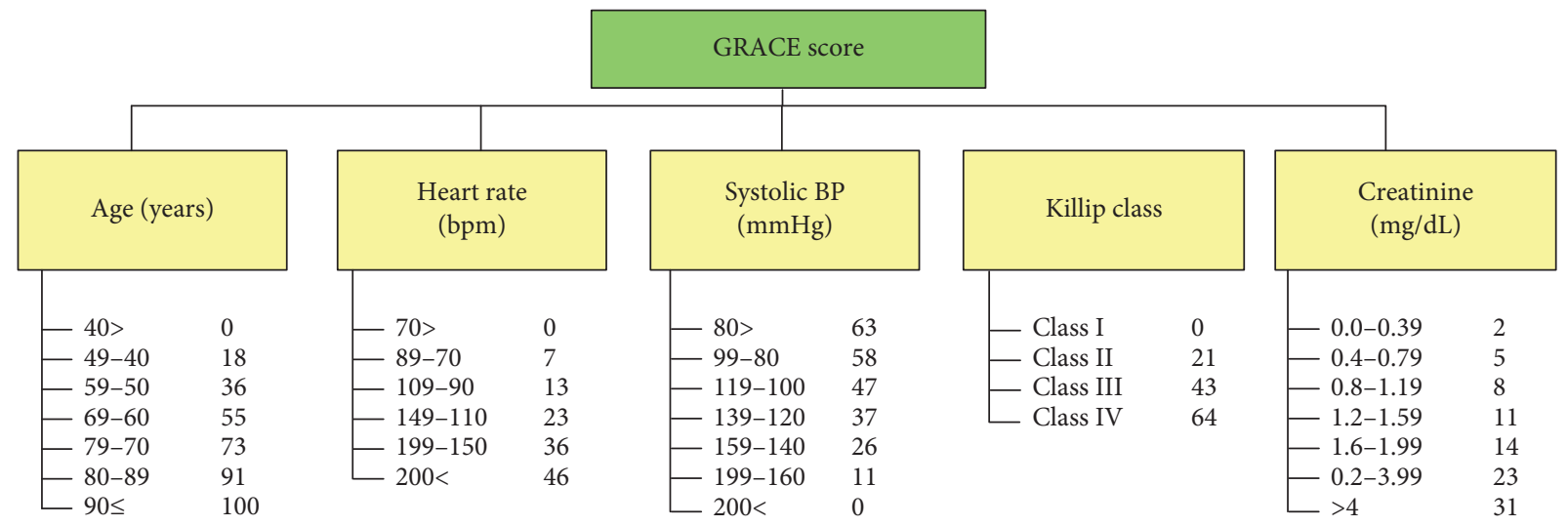

Figure 2: GRACE Score.

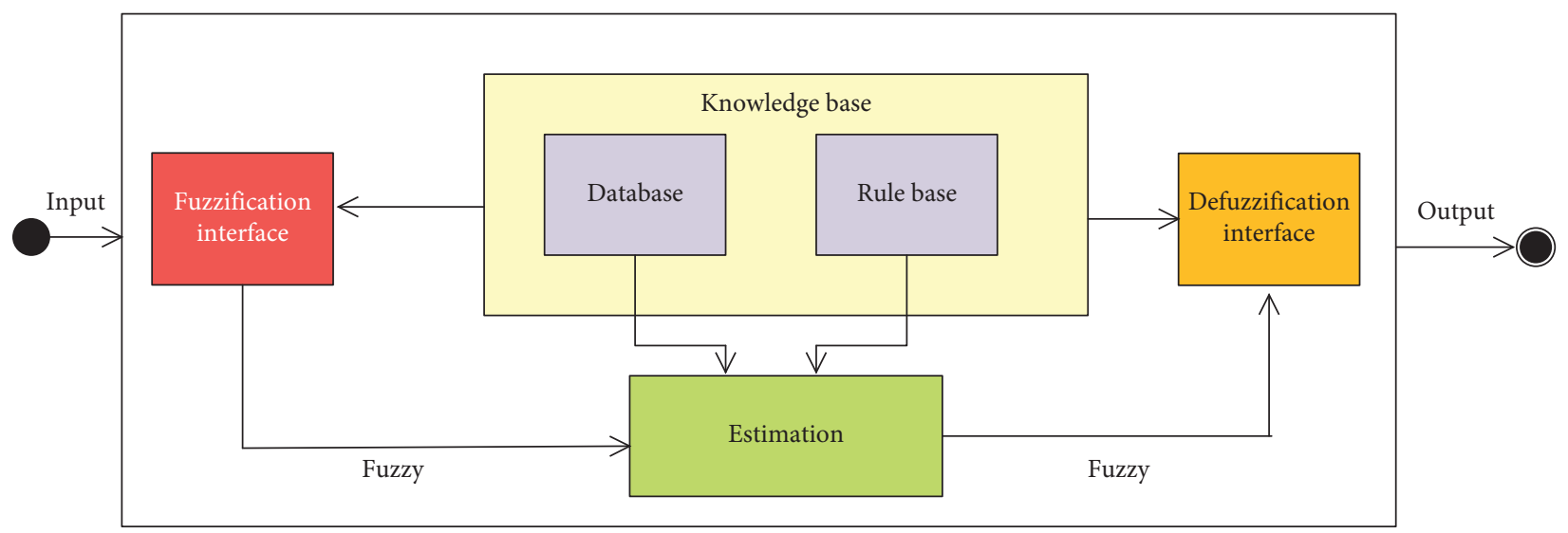

FIgURE 3: Generic process of fuzzy logic system.

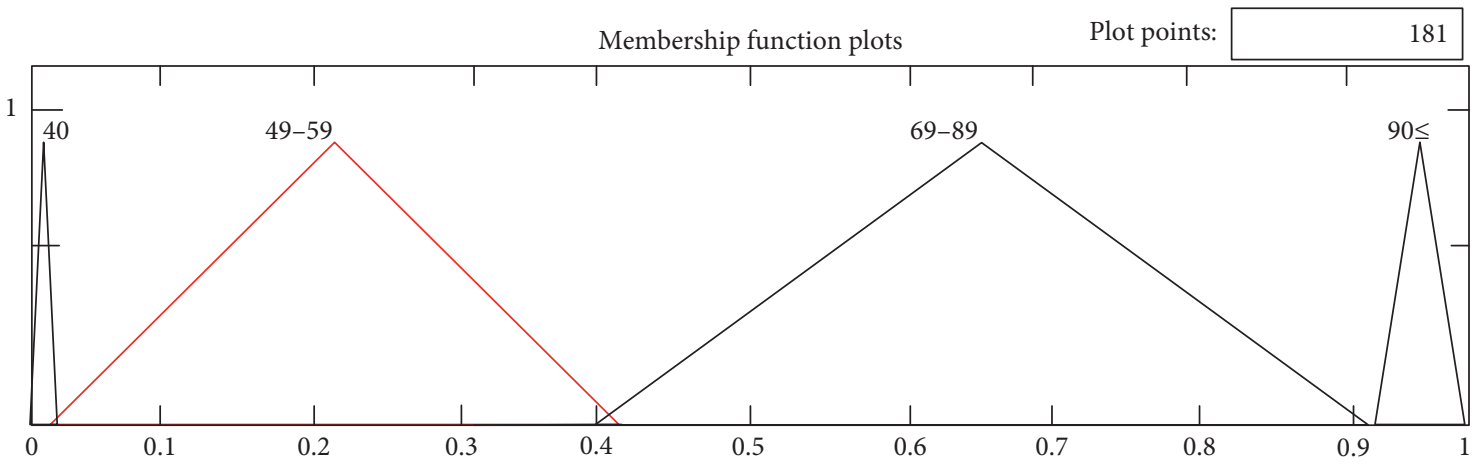

FIGURE 4: Membership functions designed for input age.

proposed system is generated in the fuzzy toolbox by using Matlab. In the figure, the initial black circles show the inputs that are the age, heart rate, systolic BP, Killip class, and creatinine, the second blank circles show the membership functions defined for the rules modeling, and the light-blue circles show the rules generated. Finally the output is shown in the figure and highlighted in black circle.

Figure 7 shows the graphical representation of the rules view obtained from membership function to design the rules and fuzzy inference system.

\section{Results and Discussions}

Once the model is designed from the membership functions for the variables of GRACE Score consisting of age, heart rate, systolic BP, Killip class, and creatinine. The fuzzy inference system of membership functions and rules are obtained. Different inputs of the same format are passed through the designed model to check the RS of the ACS. The range of the input and output values is between 0 and 1 , where 0 shows the lowest range, while 1 shows the highest 


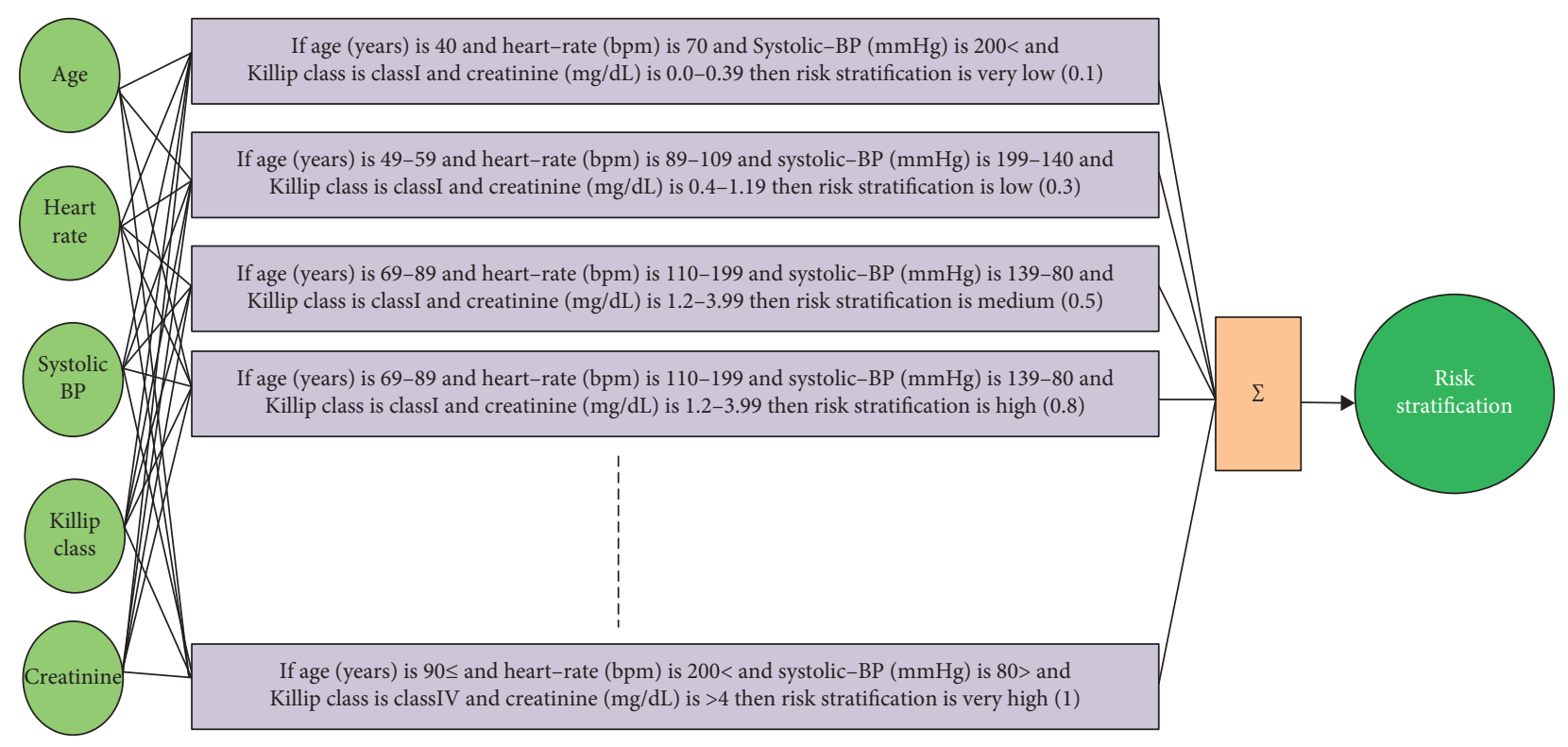

FIGURE 5: Proposed rules based system.

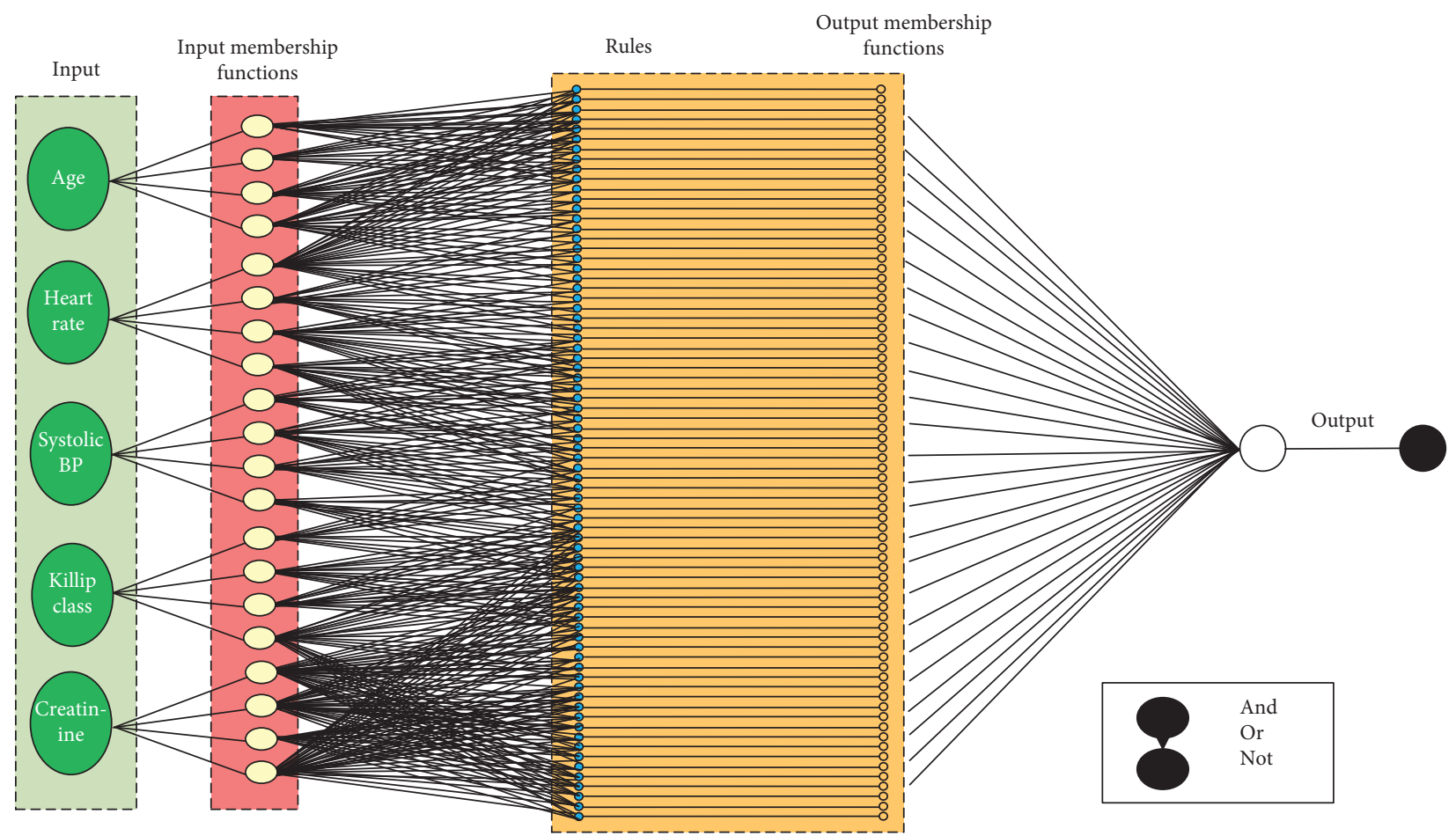

FIgURE 6: Taxonomy of the proposed inputs, membership functions, rules, and output.

range. The proposed system for risk stratification of ACS was checked and validated through a hypothetical input values. Later on, these values are confirmed from cardiologists to show the correctness of the method. The main part of this research is the design of model for RS of the ACS which was modelled in Matlab for the purpose of easiness and supporting healthcare. The values can be any digit for the features of the inputs depending on the patient's health status. Therefore, the proposed system was tested on hypothetical values to show the validity of the research. Other approaches are available as mentioned in Section 2 and Table 2, which measure the RS in ACS from different perspectives.

None of the approaches mentioned in this paper calculate the RS in ACS based on the defined criteria in complex and uncertain situation. The proposed approach can easily and effectively measure the RS in ACS. Table 3 shows the inputs that passed through the model and their output. In 


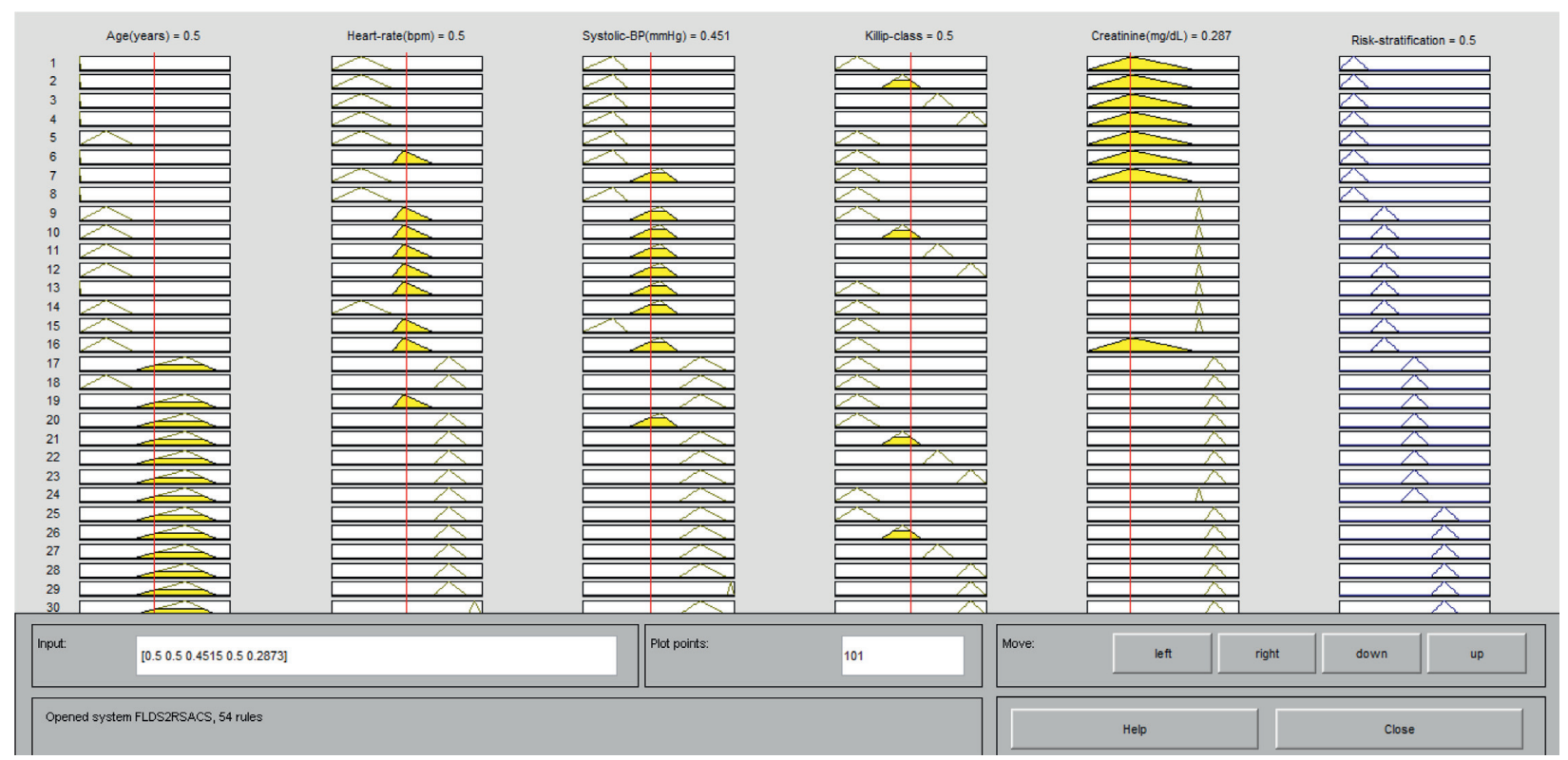

FIGURE 7: Graphical representation of rule view of the proposed system.

TABle 2: Approaches for RS in ACS.

\begin{tabular}{lcr}
\hline Reference & Method & Year \\
\hline$[42]$ & Biomarker algorithm encompassing the clinical chemistry score & 2020 \\
{$[43]$} & High-sensitivity cardiac troponin T in patients with suspected ACS & 2020 \\
{$[44]$} & Comorbidity assessment for mortality RS & 2019 \\
{$[45]$} & RS in ACS & 2018 \\
{$[46]$} & Role of cardiovascular reactions to mental stress challenge & \\
{$[43]$} & RS through high-sensitivity cardiac troponin T in patients with suspected ACS & 2020 \\
Our & Fuzzy logic based decision for RS in ACS & 2021 \\
\hline
\end{tabular}

TABLE 3: Inputs and their output of the proposed system.

\begin{tabular}{|c|c|c|c|c|c|c|}
\hline Inputs & Age & Heart rate & Systolic BP & Killip class & Creatinine & Risk stratification \\
\hline 1 & 0.1 & 0.2 & 0.1 & 0.1 & 0.2 & 0.100 \\
\hline 2 & 0.3 & 0.2 & 0.1 & 0.1 & 0.3 & 0.100 \\
\hline 3 & 0.2 & 0.3 & 0.1 & 0.2 & 0.1 & 0.100 \\
\hline 4 & 0.7 & 0.2 & 0.1 & 0.8 & 0.5 & 0.500 \\
\hline 5 & 0.8 & 0.1 & 0.4 & 0.8 & 0.9 & 0.500 \\
\hline 6 & 0.9 & 0.8 & 0.9 & 0.9 & 0.9 & 0.628 \\
\hline 7 & 0.8 & 0.8 & 0.9 & 0.9 & 0.9 & 0.626 \\
\hline 8 & 0.7 & 0.8 & 0.9 & 0.7 & 0.9 & 0.626 \\
\hline 9 & 0.9 & 0.8 & 0.9 & 0.7 & 0.9 & 0.628 \\
\hline 10 & 0.5 & 0.8 & 0.9 & 0.7 & 0.9 & 0.626 \\
\hline 11 & 0.4 & 0.8 & 0.7 & 0.7 & 0.9 & 0.628 \\
\hline 12 & 0.5 & 0.7 & 0.7 & 0.7 & 0.9 & 0.626 \\
\hline
\end{tabular}

the table, the values are considered for 12 patients with different range of inputs for the same variables of GRACE Score. The proposed system can be checked on any sample size of patients. Recently, 12 sample sizes have been considered. It can be decreased and/or increased depending on the capacity of wards or hospital.

Later on, these values are plotted diagrammatically to make it easy for the practitioners in DM process. So from the above table, the level of RS can easily be identified.

\section{Conclusion}

ACS consists of signs and symptoms to define a range of situations linked with the sudden, reduced blood flow to the heart. The heart muscles are unable to function properly due to the reduction of blood flow in the ACS. The RS in ACS aims to recognize the patients at high risk of ischemic events. On time and initial DM in healthcare has enormous prospective for enlightening the quality of care, reducing the 
cost of care, and reducing waste and error. To assist the said process, the proposed research work uses the features of GRACE Score to RS in the ACS. It will be a reliable and trustworthy method by the help of which the doctor can make early and easy DM for the patient and for detecting related disease. FL approach has been used as a tool to model the feature of GRACE score for DM in the ACS. The result of the proposed method has proved more close and authentic $\mathrm{DM}$ of the patient and can be used for advice of medicine and rest accordingly by the doctors. In the future, the proposed research can be explored to a more reliable and efficient system with large sample size to show the effectiveness and usefulness of the system for enhancement in healthcare. This will be further an enhanced step toward improvement in healthcare.

\section{Data Availability}

No data are available.

\section{Conflicts of Interest}

The authors declare no conflicts of interest.

\section{References}

[1] WHO, http://www.who.int/cardiovascular_diseases/en/, 2018.

[2] S. Goodacre and N. Calvert, "Cost effectiveness of diagnostic strategies for patients with acute, undifferentiated chest pain," Emergency Medicine Journal, vol. 20, no. 5, pp. 429-433, 2003.

[3] J. H. Pope, T. P. Aufderheide, R. Ruthazer et al., "Missed diagnoses of acute cardiac ischemia in the emergency department," New England Journal of Medicine, vol. 342, no. 16, pp. 1163-1170, 2000.

[4] K. Thygesen, J. S. Alpert, A. S. Jaffe et al., "Third universal definition of myocardial infarction," European Heart Journal, vol. 33, pp. 2551-2567, 2012.

[5] S.-Y. Lin, D.-C. Chen, C.-L. Lin et al., "Risk of acute coronary syndrome in patients with cervical spondylosis," Atherosclerosis, vol. 271, pp. 136-141, 2018.

[6] X. Liao, S. Nazir, Y. Zhou, M. Shafiq, and X. Qi, "User knowledge, data modelling and visualization- handling through fuzzy logic based approach," Complexity, vol. 2021, Article ID 6629086, 14 pages, 2021.

[7] S. Nazir, S. Shahzad, S. Mahfooz, and M. N. Jan, "Fuzzy logic based decision support system for component security evaluation," The International Arab Journal of Information Technology, vol. 15, no. 2, pp. 224-231, 2015.

[8] S. Ali, N. Ullah, M. F. Abrar, Z. Yang, and J. Huang, "Fuzzy multicriteria decision-making approach for measuring the possibility of cloud adoption for software testing," Scientific Programming, vol. 2020, Article ID 6597316, 24 pages, 2020.

[9] A. Singh and J. V. Guttag, "A comparison of non-symmetric entropy-based classification trees and support vector machine for cardiovascular risk stratification," in Proceedings of the 2011 Annual International Conference of the IEEE Engineering in Medicine and Biology Society, Boston, MA, USA, September 2011.

[10] W. A. Khalil, M. A. Saad, T. M. Mostafa, and H. E. Shairr, "Accuracy of the Canadian new risk score as in hospital prognostic score for the Egyptian patients with acute coronary syndrome," Journal of Indian College of Cardiology, vol. 8, pp. 1-6, 2018.
[11] J. F. Glatz and R. Renneberg, "Added value of H-FABP as plasma biomarker for the early evaluation of suspected acute coronary syndrome," Clinical Lipidology, vol. 9, no. 2, pp. 205-220, 2014.

[12] J. Přeček, M. Hutyra, F. Kováčik, J. Orság, and M. Táborský, "Biomarkers of renal function in prognostic stratification of patients with acute coronary syndrome," Coretvasa, vol. 60, pp. 148-154, 2018.

[13] A. V. Esbroeck and Z. Syed, "Cardiovascular risk stratification with heart rate topics," in Proceedings of the 2012Computing in Cardiology, Krakow, Poland, September 2012.

[14] S. P. Macdonald, Y. Nagree, D. M. Fatovich, H. L. Flavell, and F. Loutsky, "Comparison of two clinical scoring systems for emergency department risk stratification of suspected acute coronary syndrome," Emergency Medicine Australasia, vol. 23, no. 6, pp. 717-725, 2011.

[15] L. Cullen, J. Greenslade, M. Than et al., "Performance of risk stratification for acute coronary syndrome with two-hour sensitive troponin assay results," Heart Lung \& Circulation, vol. 23, no. 5, pp. 428-434, 2014.

[16] D. Corcoran, P. Grant, and C. Berry, "Risk stratification in non-ST elevation acute coronary syndromes: risk scores, biomarkers and clinical judgment," IJC Heart \& Vasculature, vol. 8, pp. 131-137, 2015.

[17] Z. Syed, B. Scirica, C. Stultz, and J. Guttag, "Risk-stratification following acute coronary syndromes using a novel electrocardiographic technique to measure variability in morphology," in Proceedings of the 2018 Computers in Cardiology, Bologna, Italy, September 2008.

[18] B. Doyle, D. Fitzsimons, P. McKeown, and T. McAloon, "Understanding dietary decision-making in patients attending a secondary prevention clinic following myocardial infarction," Journal of Clinical Nursing, vol. 21, no. 1-2, pp. 32-41, 2011.

[19] C. Dellas, J. L. Lobo, A. Rivas et al., "Risk stratification of acute pulmonary embolism based on clinical parameters, H-FABP and multidetector CT, International Journal of Cardiology, vol. 265, pp. 223-228, 2018.

[20] F. J. Pastor-Perez, F. Soria-Arcos, P. Morillas-Blasco et al., "Additive value of diabetes and peripheral arterial disease in the risk stratification of patients admitted after an acute coronary syndrome: a subanalysis of the PAMISCA Study," International Journal of Clinical Practice, vol. 63, no. 9, pp. 1314-1319, 2009.

[21] A. P. Hill, M. D. Perry, N. Abi-Gerges et al., "Computational cardiology and risk stratification for sudden cardiac death: one of the grand challenges for cardiology in the 21st century," The Journal of Physiology, vol. 594, no. 23, pp. 6893-6908, 2016.

[22] A. T. Timóteo, S. Aguiar Rosa, M. Afonso Nogueira, A. Belo, and R. Cruz Ferreira, "ProACS risk score: an early and simple score for risk stratification of patients with acute coronary syndromes," Revista Portuguesa de Cardiologia, vol. 36, no. 2, pp. 77-83, 2017.

[23] A. J. Mirza, A. Y. Taha, and B. R. Khdhir, "Risk factors for acute coronary syndrome in patients below the age of 40 years," The Egyptian Heart Journal, vol. 70, pp. 1-3, 2018.

[24] T. Pezawas, A. Diedrich, D. Robertson et al., "Risk of arrhythmic death in ischemic heart disease: a prospective, controlled, observer-blind risk stratification over 10 years," European Journal of Clinical Investigation, vol. 47, no. 3, pp. 231-240, 2017.

[25] A. Timmis, "CORONARY DISEASE-acute coronary syndromes: risk stratification,” Heart, vol. 84, 2000. 
[26] S. P. Macdonald and Y. Nagree, "Rapid risk stratification in suspected acute coronary syndrome using serial multiple cardiac biomarkers: a pilot study," Emergency Medicine Australasia, vol. 20, no. 5, pp. 403-409, 2008.

[27] L. Paiva, S. Barra, and L. Gonçalves, "Risk stratification in acute coronary syndromes: g," Revista Portuguesa de Cardiologia, vol. 36, no. 9, pp. 677-679, 2017.

[28] D. Ferreira, "Risk stratification after acute coronary syndromes: scores, scores and yet another score," Revista Portuguesa de Cardiologia, vol. 36, no. 2, pp. 85-87, 2017.

[29] I. Y. Gong and A. T. Yan, "A new risk stratification tool for women with acute coronary syndrome," International Journal of Cardiology, vol. 259, pp. 53-54, 2018.

[30] K. A. Eagle, M. J. Lim, O. H. Dabbous et al., "A validated prediction model for all forms of acute coronary syndrome," Jama, vol. 291, no. 22, pp. 2727-2733, 2004.

[31] C. B. Granger, R. J. Goldberg, O. Dabbous et al., "Predictors of hospital mortality in the global registry of acute coronary events," Archives of Internal Medicine, vol. 163, no. 19, pp. 2345-2353, 2003.

[32] L. A. Zadeh, "Fuzzy logic," Computer, vol. 21, no. 4, pp. 83-93, 1988.

[33] Fuzzy Logic Tool Box ${ }^{T M} 2$ User's Guide, The MathWorks, Inc. 3 Apple Hill Drive Natick, Natick, MA, USA, 2010, https://ww2. mathworks.cn/help/fuzzy/index.html?requestedDomain $=\mathrm{cn}$.

[34] J. Yen and R. Langari, Fuzzy Logic: Intelligence, Control and Information, Prentice-Hall, Hoboken, NJ, USA, 1st edition, 1999.

[35] S. Nazir, M. A. Khan, S. Anwar, H. Khan, and M. Nazir, "A novel fuzzy logic based software component selection modeling," in Proceedings of the2012 International Conference on Information Science and Applications, pp. 1-6, IEEE, Suwon, Korea, May 2012.

[36] S. Nazir and M. Nazir, "Comparisons of membership functions for fuzzy rules," Vawkum Transactions on Computer Sciences, vol. 3, no. 1, pp. 10-14, 2014, http://vfast.org/index. $\mathrm{php} / \mathrm{VTCS}$.

[37] S. Nazir, S. Shahzad, I. Zada, and H. Khan, "Evaluation of software birthmarks using fuzzy analytic hierarchy process," in Proceedings of the Fourth International Multi-Topic Conference, pp. 171-175, Jamshoro, Pakistan, February 2015.

[38] M. A. B. Messaoud, A. Bouzid, and N. Ellouze, "A new biologically inspired fuzzy expert system-based voiced/unvoiced decision algorithm for speech enhancement," Cognitive Computation, vol. 8, no. 3, pp. 478-493, 2016.

[39] P. Liu and X. Zhang, "A novel picture fuzzy linguistic aggregation operator and its application to group decisionmaking," Cognitive Computtation, vol. 100, no. 2, 2018.

[40] N. Liu, J. T. Sakamoto, J. Cao et al., "Ensemble-based risk scoring with extreme learning machine for prediction of adverse cardiac events," Cognitive Computation, vol. 9, no. 4, pp. 545-554, 2017.

[41] C. W. Hamm, J.-P. Bassand, S. Agewall et al., "ESC Guidelines for the management of acute coronary syndromes in patients presenting without persistent ST-segment elevation," European Heart Journal, vol. 32, pp. 2999-3054, 2011.

[42] P. A. Kavsak, S. E. Mondoux, and J. Ma, "Comparison of two biomarker only algorithms for early risk stratification in patients with suspected acute coronary syndrome," International Journal of Cardiology, vol. 319, pp. 140-143, 2020.

[43] A. R. Chapman, D. Sandeman, A. V. Ferry et al., "Risk stratification using high-sensitivity cardiac troponin $\mathrm{T}$ in patients with suspected acute coronary syndrome," Journal of the American College of Cardiology, vol. 75, no. 8, pp. 985-987, 2020.

[44] J. Sanchis, M. Soler, J Núñez et al., "Comorbidity assessment for mortality risk stratification in elderly patients with acute coronary syndrome," European Journal of Internal Medicine, vol. 62, pp. 48-53, 2019.

[45] J. Ferreira, "Risk stratification in acute coronary syndromes: when less is more," Revista Portuguesa de Cardiologia, vol. 37, no. 11, pp. 921-922, 2018.

[46] J. Gecaite, J. Brozaitiene, and N. Mickuviene, “P.515 risk stratification in patients after acute coronary syndrome: the role of cardiovascular reactions to mental stress challenge," European Neuropsychopharmacology, vol. 29, pp. S363-S364, 2019. 